\title{
PROPOSTA DE ÍNDICE PARA AVALIAÇÃO DE SITUAÇÃO DE VULNERABILIDADE SOCIAL À COVID-19
}

\section{PROPOSED INDEX TO ASSESS SITUATION OF SOCIAL VULNERABILITY TO COVID-19}

\author{
Juliana Ramalho Barros \\ Professora Doutora, Universidade Federal de Goiás. \\ juliana@ufg.br
}

Thamy Barbara Gioia

Doutoranda, Programa de Pós-graduação em Geografia pela Universidade Federal de Goiás. thamygioia@gmail.com

Hérika Silva Vasques

Doutoranda, Programa de Pós-graduação em Geografia pela Universidade Federal de Goiás. hrkgeo@hotmail.com

\begin{abstract}
RESUMO
O processo saúde-doença é permeado por fatores que vão além da susceptibilidade genética e biológica, mas também por variáveis ligadas às condições sociais e econômicas que podem ser expressas em situações de vulnerabilidade em saúde. O cenário de expansão da COVID-19 no Brasil tem demonstrado como as desigualdades sociais repercutem nesse processo saúde-doença, de modo que, avaliar tais disparidades pode oferecer suporte ao enfrentamento da doença no País. O objetivo do presente artigo foi estabelecer um índice para avaliação da situação de vulnerabilidade social à COVID-19. A partir da seleção de 12 variáveis, a modelagem consistiu na identificação das mais preditivas à ocorrência da COVID-19 no Estado de Goiás e no Distrito Federal. Para isso, foram testados dois algoritmos de machine learning: Random Forest e XGBoost. Os resultados indicaram como mais preditivas as variáveis: condições de renda, total de internações por doenças classificadas como mais vulneráveis e porcentagem da população em condições de trabalho informal. Diante disso, aproximadamente $23 \%$ dos municípios foram classificados em alta a muito alta vulnerabilidade.
\end{abstract}

Palavras-chave: Índice de Vulnerabilidade Social. algoritmos de machine learning COVID19. Goiás.

\begin{abstract}
The health-disease process encompasses factors beyond genetic and biological susceptibility, but also includes variables linked to social and economic conditions that can lead to health vulnerability. The expanding situation of COVID-19 in Brazil has demonstrated how social inequalities affect this health-disease process; thus, evaluating such disparities can help the country confront the disease. The objective of this article was to establish an index to assess the situation of social vulnerability to COVID-19. From the 12 selected variables, the modeling identified those the predicted the occurrence of COVID-19 in the State of Goiás and the Federal District. For this, two machine learning algorithms were tested: Random Forest and XGBoost. The results indicated the most predictive variables were income status, the total hospitalizations for ailments classified as very vulnerable, and the percentage of the population working informally. Therefore, approximately $23 \%$ of the municipalities were classified with high to very high vulnerability.
\end{abstract}

Keywords: Social Vulnerability Index. Machine learning algorithms. COVID-19. Goiás.

Recebido em: 07/05/2020

Aceito para publicação em: 21/05/2020. 


\section{INTRODUÇÃO}

A contemporaneidade é marcada por um mundo globalizado e urbanizado, em que os grandes fluxos de informação, comunicação, pessoas e mercadorias estabelecem a dinâmica global. Esse processo contribuiu significativamente para a maior concentração das pessoas nas cidades e, consequentemente, para mudanças dos padrões de morbidade e mortalidade.

A emergência da pandemia da COVID-19 evidencia que o processo saúde-doença deve ser entendido em diferentes escalas - global, regional e local, considerando-se que, no mundo globalizado, ampliam-se as desigualdades sociais e tempo e espaço assumem novas dimensões no que diz respeito aos fluxos, aos usos e às forças hegemônicas atuantes. Um dos resultados disso é ter-se grande parcela da população vivendo em situações de vulnerabilidade e, conforme sinaliza Santana (2016, p. 29), "[...] existe uma associação entre a ocorrência da doença e os fatores sociais, econômicos e culturais do local onde os indivíduos passam a maior parte do seu tempo".

O conceito de vulnerabilidade de Czeresnia e Oviedo (2015) indica que a essa está ligada as relações que delimitam a habilidade de atuação dos indivíduos ou dos grupos e que afastam o exercício de direitos. Assim, entendem que uma das maneiras de compreender a suscetibilidade das pessoas em adoecer ou morrer está relacionada à compreensão dos determinantes sociais envolvidos.

O novo coronavírus surgiu na cidade de Wuhan, província de Hubei, na China, em dezembro de 2019, e foi denominado pela Organização Mundial da Saúde - OMS como COVID-19. Vírus da família coronavírus, como a síndrome respiratória aguda grave - SARS e a síndrome respiratória do Oriente Médio - MERS, na maioria das vezes desenvolve sintomas leves, porém, em alguns casos pode levar o paciente a óbito (SOHRABI, et al. 2020).

No Brasil, a doença já atinge mais de 125.000 pessoas e o registro de óbitos já ultrapassa 8.000 . Dentre as regiões do País, o Sudeste é a mais afetada, concentrando aproximadamente $48 \%$ do total de casos registrados, seguindo da região Nordeste, com cerca de $30 \%$ dos casos. A região Centro-Oeste apresenta o menor número de casos e representa uma parcela de $3 \%$ do total $^{2}$ (BRASIL, 2020).

Desta forma, o objetivo deste trabalho foi estabelecer um índice para avaliação da situação de vulnerabilidade social à COVID-19 - IVSc. Trata-se de um estudo experimental e preliminar, considerando-se os poucos dados e informações disponíveis, mas que pretende colaborar para o avanço das pesquisas na temática em questão, buscando, principalmente, auxiliar os setores públicos na gestão de ações para enfrentamento da doença.

\section{MATERIAIS E MÉTODOS}

\section{Área de Estudo}

Como área de estudo delimitou-se o Estado de Goiás, com seus 246 municípios, e o Distrito Federal (Figura 1). Localizados na região Centro-Oeste do Brasil, Goiás possui área de $340.111,38 \mathrm{~km}^{2}$, população estimada de 7.018 .354 pessoas e densidade demográfica de 17,65 hab. $/ \mathrm{km}^{2}$ (IBGE, 2019), enquanto o Distrito Federal ocupa uma área de $5.760,783 \mathrm{~km}^{2}$, tem uma população estimada em 3.015.268 pessoas e densidade demográfica de 444,07 hab./km² (IBGE, 2019).

Ambas as unidades da federação, apresentam Índice de Desenvolvimento Humano - IDH considerado elevado, de 0,735 e 0,824 o Estado de Goiás e o Distrito Federal, respectivamente (IBGE, 2010). Apesar disso, tanto Goiás como o Distrito Federal são caracterizados por enormes disparidades socioeconômicas entre os municípios e regiões administrativas que os compõem.

\footnotetext{
${ }^{2}$ Estes dados referem-se à data de 07 de maio de 2020 e podem ser visualizados no site oficial do Ministério da Saúde - Coronavírus Brasil: https://covid.saude.gov.br/. 
Figura 1 - Mapa de localização do Estado de Goiás e do Distrito Federal

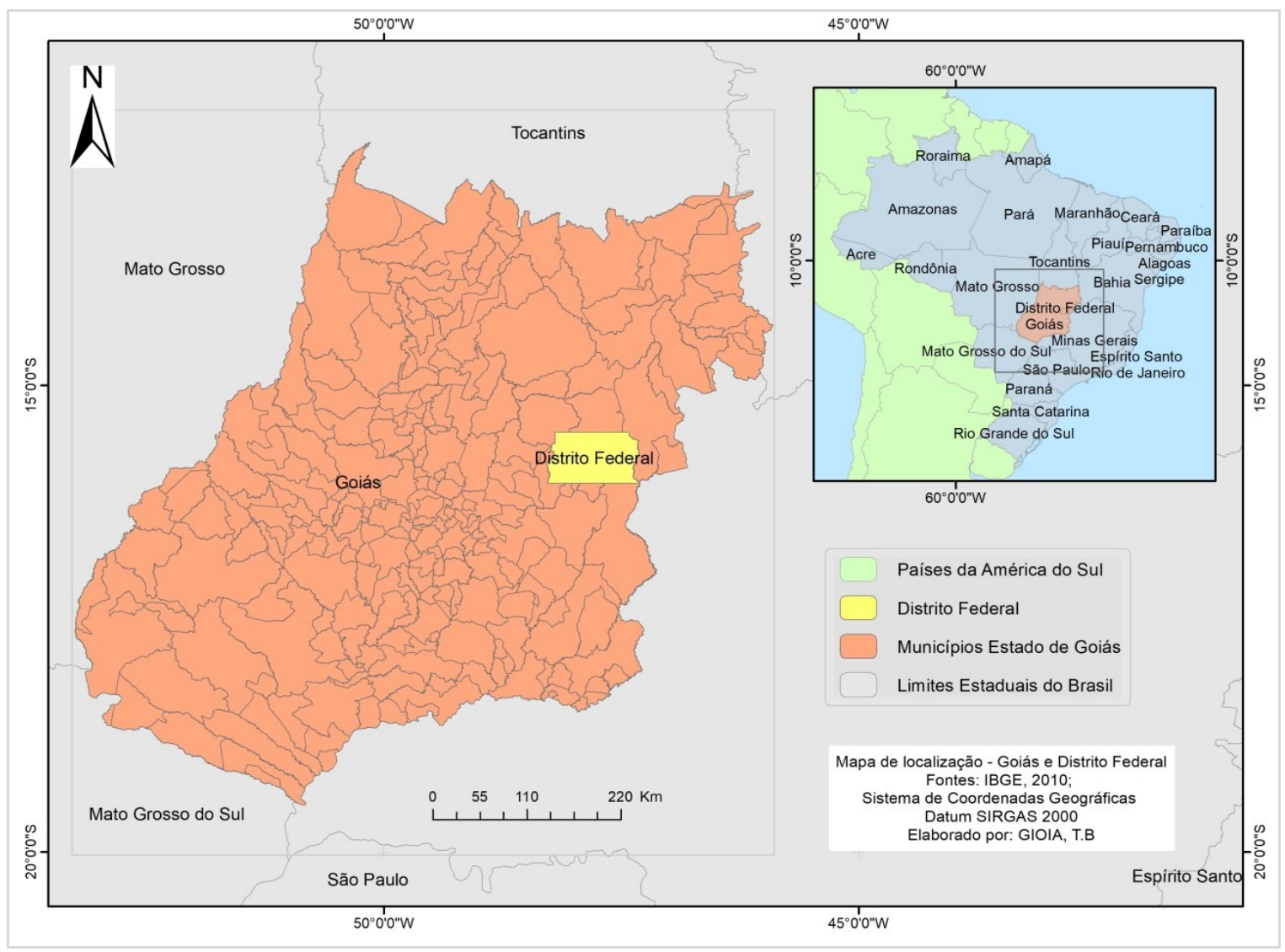

Elaborado por GIOIA, T. B., 2020.

\section{Materiais}

O Índice de Vulnerabilidade Social - IVSc foi estabelecido com base em dados secundários disponibilizados em bases públicas: Instituto Brasileiro de Geografia e Estatística - IBGE: https://sidra.ibge.gov.br; Departamento de Informática do SUS - DATASUS: https://datasus.saude.gov.br/informacoes-de-saude-tabnet/; Ministério do Trabalho - MTE: http://trabalho.gov.br/rais; e Secretaria de Saúde do Estado de Goiás, por meio da base oficial do Laboratório de Processamento de Imagens e Geoprocessamento - LAPIG/Plataforma COVID GOIAS: https://covidgoias.ufg.br/. Acrescidas as bases estatísticas, utilizou-se das bases cartográficas digitais em formato vetorial disponíveis em https://www.ibge.gov.br/geociencias/downloads-geociencias.html, para posterior espacialização dos resultados.

No Quadro 1 são apresentadas as variáveis utilizadas para composição do IVSc: 
Quadro 1 - Variáveis utilizadas no estudo, suas fontes oficiais e referências para aplicação metodológica.

\begin{tabular}{|c|c|c|}
\hline Cód. & Descrição & Fonte de dados \\
\hline V1 & Razão morador por domicílio. & IBGE (2010) \\
\hline V2 & \% de população urbana. & IBGE (2010) \\
\hline V3 & \% população acima de 60 anos. & IBGE (2010) \\
\hline V4 & $\begin{array}{l}\text { \% de domicílios em situação inadequada de abastecimento de água - } \\
\text { todos os domicílios que não recebam água por rede pública ou que } \\
\text { não tenham poço na propriedade. }\end{array}$ & IBGE (2010) \\
\hline V5 & \% de população negra, parda, indígena. & IBGE (2010) \\
\hline V6 & $\begin{array}{l}\text { \% de população em situação de trabalho informal - obtido a partir do } \\
\text { total de população em emprego formal pelo total da população } \\
\text { economicamente ativa. }\end{array}$ & $\begin{array}{l}\text { MTE/RAIS } \\
\text { IBGE (2010) }\end{array}$ \\
\hline v7 & $\begin{array}{l}\text { \% de domicílios com renda de até dois salários mínimos incluindo } \\
\text { domicílios sem renda. }\end{array}$ & IBGE (2010) \\
\hline V8 & $\begin{array}{l}\text { Total de internações por doenças: tuberculose, pneumonia, doenças } \\
\text { respiratórias, hipertensão, diabetes, demais doenças cardiovasculares, } \\
\text { insuficiência renal. }\end{array}$ & DATASUS (Jan/Fev, 2020) \\
\hline v9 & $\begin{array}{l}\text { Proximidade das principais rodovias do Estado de Goiás: BR153 e } \\
060 \text {. }\end{array}$ & IBGE/REGIC (2007) \\
\hline V10 & Condição de centralidade. & IBGE/REGIC (2007) \\
\hline V11 & Total de leitos com UTI a cada 10.000 habitantes. & DATASUS (Jan/Fev, 2020) \\
\hline D & $\begin{array}{l}\text { Distâncias mínimas do município mais próximo onde tenham sido } \\
\text { registradas casos de COVID-19 na primeira semana de março. }\end{array}$ & $\begin{array}{l}\text { Obtidos por meio de } \\
\text { ferramentas de } \\
\text { geoprocessamento }\end{array}$ \\
\hline C & Total de casos confirmados de COVID-19 até a data de 26/04/2020. & $\begin{array}{l}\text { Secretária de Saúde do } \\
\text { Estado de Goiás/LAPIG }\end{array}$ \\
\hline
\end{tabular}

\section{Metodologia}

Para modelagem do IVSc, as variáveis destacadas no Quadro 1 foram organizadas em uma única planilha e concatenadas em uma base com os respectivos códigos municipais para que os resultados pudessem ser espacializados posteriormente. A cada variável foi atribuído um código para facilitar a apresentação dos resultados (Quadro 1).

A modelagem consistiu na identificação das variáveis mais preditivas à ocorrência de casos de COVID-19 nos municípios do Estado de Goiás e no Distrito Federal, considerando o aumento de número de casos nas oito semanas iniciais da primeira ocorrência. Para isso, foram testados dois algoritmos de aprendizado em máquina (machine learning) baseados em árvore de decisão: Random Forest (BREIMAN; CLUTER, 2001), que trabalha a partir da combinação de árvores de decisão independentes e o XGBoost (CHEN; GUESTRIN, 2016), por meio da combinação de árvores de decisão sequenciais. Os algoritmos de árvore de decisão têm por objetivo dividir um conjunto de variáveis em subvariáveis. Em casos de árvores de decisão para regressão, são considerados os valores médios dos conjuntos de amostras de cada variável.

Como técnica de calibração e validação dos modelos, utilizou-se da validação cruzada $k$-fold, sendo $\mathrm{k}=10$. Para definição do algoritmo de melhor desempenho levou-se em conta os resultados de coeficiente de determinação $\left(R^{2}\right)$ e da raiz quadrada do erro quadrático médio - RMSE. Para realização destes procedimentos foram utilizados scripts específicos aplicados no software de estatística $R$ versão 3.5 .1 com base no pacote Classification and Regression Training - CARET (KUHN, 2017).

Para cada modelo foi computada a importância de cada variável (GRÖMPING, 2015) na estimativa da ocorrência de casos de COVID-19 nos municípios do Estado de Goiás e no Distrito Federal. Os níveis de importância, normalizados em porcentagem, foram convertidos proporcionalmente em pesos para cada variável. O IVSc foi então estabelecido por:

$$
\mathrm{IVS}=\mathrm{V} 1 \mathrm{p} 1+\mathrm{V} 2 \mathrm{p} 2
$$


Vx - Corresponde ao valor de cada variável preditiva já normalizada;

$p$ - Peso atribuído a partir da conversão proporcional relativo ao nível de importância de cada variável indicado pelo resultado do algoritmo de melhor desempenho;

As variáveis preditivas foram normalizadas de 0 a 1 antes de serem aplicadas na equação IVSc. Tal procedimento foi realizado para que fosse possível inverter os valores de distância e total de leitos por habitantes, considerando que nestes casos quanto menor o valor, maior a vulnerabilidade. A normalização foi realizada por meia da Equação 2:

variảvel normalizada $=\frac{\text { valor bruto-valor mínimo }}{\text { valor máximo-valor mínimo }}$

$\mathrm{Na}$ sequência, a planilha com os resultados do IVSc foi concatenada a malha municipal e espacializada por níveis de desvio padrão conforme a seguinte classificação:

Baixa: municípios com valores de IVS inferiores ao IVS médio;

Média: municípios com valores do IVS com 0,5 +/- da média de desvio padrão;

$>$ Elevada: municípios com valores acima do IVS médio até o limite de 1,5 de desvio padrão;

> Muito elevada: municípios com valores acima de 1,5 de desvio padrão;

Estes procedimentos foram realizados no software QGIS versão 3.4.5.

\section{RESULTADOS E DISCUSSÕES}

Os valores de $R^{2}$ para os dois algoritmos foram próximos de 0,60 , ou seja, ambos os modelos foram capazes de inferir cerca de $60 \%$ do número de casos de COVID-19 nas oito primeiras semanas de registros. Sendo assim, levou-se em consideração os valores de RMSE para a decisão sobre qual o melhor modelo e, neste caso, o modelo Random Forest com RMSE 17,72 apontou melhor desempenho, enquanto o XGBoost apresentou um RMSE de 18,05.

Com base na proposta metodológica apresentada, escolhido o modelo Random Forest como de melhor desempenho, foram selecionadas 11 variáveis mais preditivas à ocorrência da COVID-19 nos municípios com registros confirmados. Na Figura 2 é possível observar que, dentre as variáveis mais preditivas, as de maior destaque (4 primeiras) foram: V7 - \% de domicílios com renda de até dois salários mínimos, V8 - Total de internações por doenças mais vulneráveis, V6 \% de população em situação de trabalho informal e D - Distâncias mínimas do município mais próximo onde tenham sido registradas casos de COVID-19 na primeira semana de março. A variável V9 - Proximidade das principais rodovias do Estado de Goiás, não apresentou valores significativos se comparada às demais variáveis testadas.

No Mapa da Figura 3, apresentam-se os resultados do IVSc para os municípios Goianos e para o Distrito Federal. 74 municípios mais o Distrito Federal, ou seja, cerca de $30 \%$ do total das unidades territoriais ${ }^{3}$ analisadas, foram classificados em vulnerabilidade baixa (em amarelo no mapa). No que diz respeito aos municípios goianos, estudos anteriores (IMB, 2018) já indicavam as boas condições socioeconômicas destes municípios quando comparados aos demais do Estado, destacando-se Jataí, Serranópolis, Ouvidor, Três Ranchos, Itumbiara e Catalão. Nesse grupo, a taxa média de urbanização foi de $78,6 \%$, a porcentagem de população negra, parda e indígena foi a mais baixa, $52 \%$, e cerca de $60 \%$ da população exercem atividades informais.

\footnotetext{
3 Considerando que o Distrito Federal não se constitui como unidade municipal e sim território autônomo, utilizaremos o termo "unidades territoriais" quando o objetivo for contemplar no discurso tanto o Distrito Federal como os municípios do Estado de Goiás. 
Figura 2 - Níveis de importância das variáveis preditivas aos municípios com casos de COVID -19.

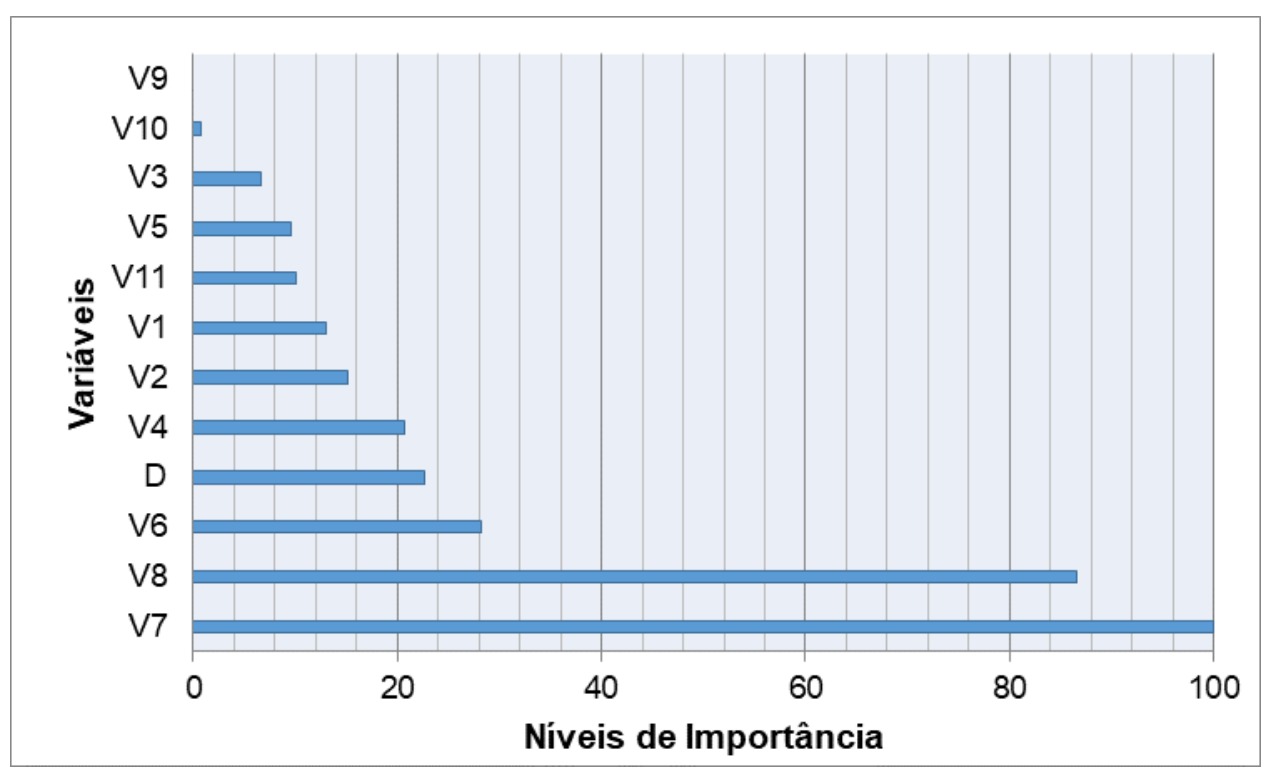

Elaborado por GIOIA, T. B., 2020.

Figura 3 - Mapa de IVSc - Municípios de Goiás e Distrito Federal

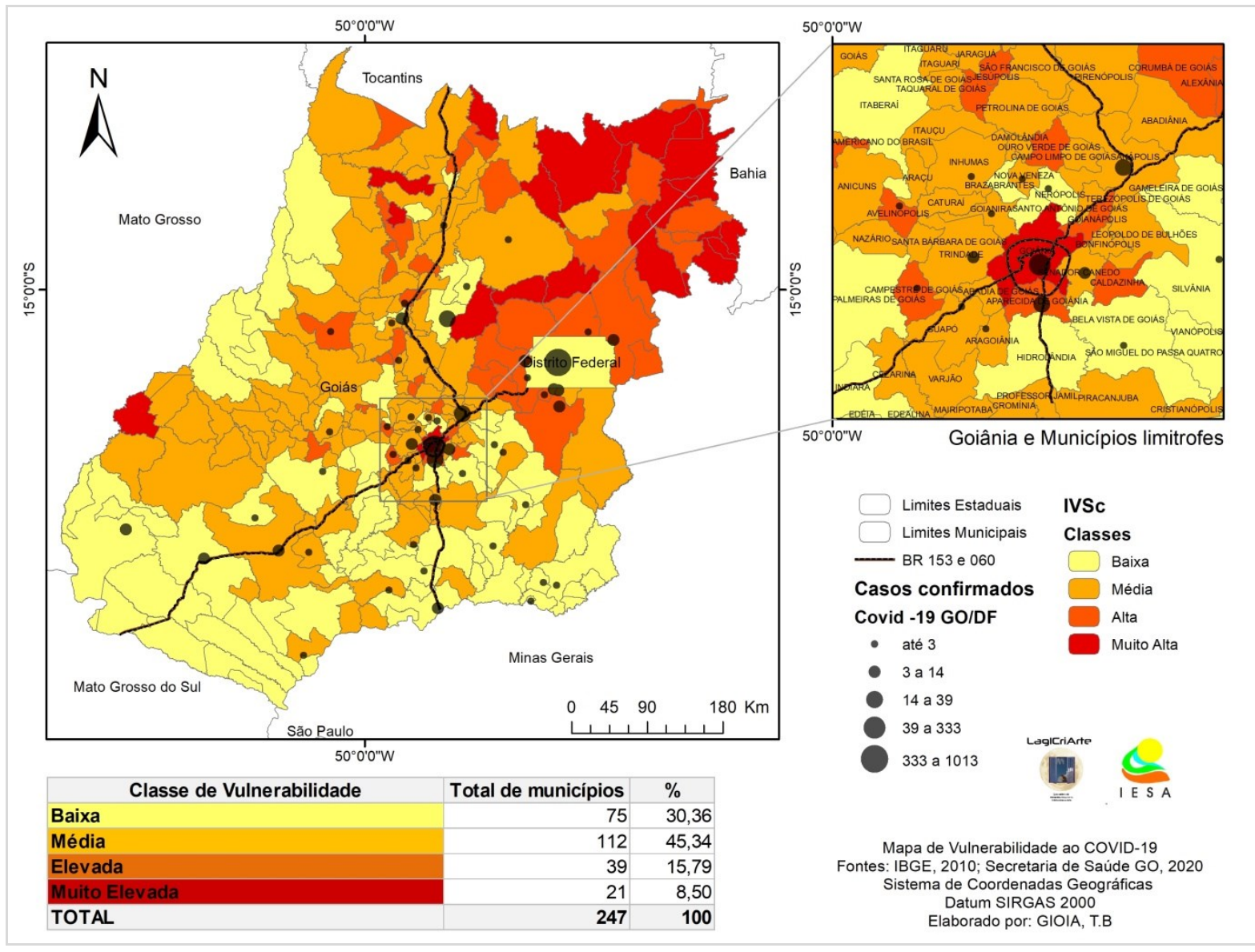

Elaborado por GIOIA, T. B., 2020. 
$\mathrm{Na}$ classe de média vulnerabilidade, foram classificados 112 municípios goianos, o que equivale a aproximadamente $45 \%$ do total. Nesses municípios destaca-se a variável V3, porcentagem de população acima de 60 anos $^{4}(12,5 \%)$ e a variável referente às atividades informais, visto que, em média, $77,3 \%$ da população desses municípios exercem trabalho informal. Ainda, $24,6 \%$ dos domicílios foram classificados com renda de até 2 salários mínimos, variável que também inclui domicílios sem rendimento. De acordo com estudos referentes a epidemias anteriores, como o de Bucchianeri (2010), as desigualdades sociais mostram-se decisivas para a taxa de severidade e transmissão de infecções respiratórias, o que aponta para a forte relação entre a baixa renda a vulnerabilidade das populações dos municípios analisados.

O município de Baliza mostra-se como uma exceção entre aqueles do Noroeste Goiano, pois apresentou um índice de vulnerabilidade multo alto. Pesquisa realizada pelo Instituto Mauro Borges de Estatística e Estudos Socioeconômicos - IMB - (2018, p. 20), já havia identificado o município como exceção aos demais da região. O município foi classificado no Grupo 2 de vulnerabilidade e, de acordo com o estudo, no município "a estrutura de oportunidades oferecida pelo mercado de trabalho também é bastante preocupante. Aqui se encontra o menor percentual de trabalhadores com carteira assinada [...]", da mesma forma, a variável percentual da população em trabalho informal foi a que mais se destacou para classificação deste município na categoria de vulnerabilidade muito alta para o IVSc, cerca de $90 \%$ da população encontra-se em situação de trabalho informal no município.

$15,78 \%$ dos municípios (39 do total) foram classificados em situação de vulnerabilidade alta, sendo boa parte deles localizados na região central e mais a noroeste do estado de Goiás. Chama atenção a alta concentração de municípios nesta categoria localizados no entorno do Distrito Federal e na região do extremo nordeste do Estado de Goiás. A taxa de urbanização nesses municípios é de $74 \%$, a deficiência no sistema de abastecimento de água é de $8,18 \%$, o trabalho informal atinge $80 \%$ da população economicamente ativa e cerca de $30 \%$ dos domicílios classificam-se na condição de renda vulnerável. Fazem parte deste grupo os municípios de Formosa, Nova Roma, Planaltina, Posse e Campos Belos.

Observa-se que apenas 21 (8,5\%) municípios estão na faixa IVSc muito alta, representando, assim, o grupo mais suscetível. Nessas localidades, observam-se a menor taxa de urbanização registrada $(53,17 \%)$, o maior quantitativo de deficiência de abastecimento de água $(23,01 \%)$ e total de população negra, parda e indígena $(72,61 \%)$. Também se destacam por apresentarem uma grande parcela da população ocupando postos de trabalho informal $(81,58 \%)$ e com cerca de $40 \%$ dos domicílios com baixos rendimentos (até 2 salários mínimos). Representa esse grupo os municípios de Cavalcante, Teresina de Goiás, Mimoso de Goiás e Água Fria de Goiás.

Ao contrário do que foi observado no trabalho elaborado pelo IMB (2018), Goiânia se insere na classe de muito alta vulnerabilidade, mesmo apresentando os melhores índices no que diz respeito ao abastecimento de água, população economicamente ativa em condições formais de trabalho, e o menor índice de população com renda classificada como vulnerável se comparado às demais do Estado de Goiás. Goiânia apresentou o maior índice de internações por doenças consideradas mais susceptíveis à COVID-195, uma população urbana de mais de $98 \%$, cerca de $50 \%$ da população é negra, parda ou indígena, além de ser cidade de alta centralidade por onde passa as principais rodovias do Estado. As causas desse resultado precisam ser mais bem analisadas, contudo, o grande fluxo de pessoas e a polarização que a cidade exerce em relação ao restante do estado e mesmo a outras regiões do País podem ser fatores causais para a elevada vulnerabilidade encontrada.

Conforme a relação de casos confirmados até o dia 26/04/2020, a capital federal apresentava o maior número de casos acumulados - 1013. No entanto, o Distrito Federal foi classificado com baixa vulnerabilidade. De fato, se comparado aos demais municípios de Goiás em relação aos indicadores expressos pelas variáveis analisadas, o Distrito Federal apresenta melhores condições. Contudo, é importante destacar que Brasília apresentou os primeiros registros de casos, indicando que se trata de um centro de dispersão da doença. O IVSc indica, ainda, que os municípios do Estado de Goiás, limítrofes a Brasília, encontram-se em vulnerabilidade social muito alta, indicando a fragilidade desses

\footnotetext{
${ }^{4}$ Conforme as orientações da OMS/OPAS, adultos maiores de 60 anos e pessoas com doenças pré-existentes, como diabetes e cardiopatias tem maior risco de adquirir a COVID-19. Para mais informações acessar a folha técnica disponível https://www.paho.org/bra/index.php?option=com content\&view=article\&id=6101: covid19\&ltemid=875
} 
municípios caso venham a ter aumento no número de casos de COVID-19. Este cenário repete-se entre os municípios de Goianésia e Barro Alto, que foram classificados com baixa vulnerabilidade, mas já apresentavam quantitativo de casos significativos, 29 no total (26/04/2020), e o município de Vila Propício, classificado em vulnerabilidade muito alta, que até a data de elaboração do estudo não apresentava casos confirmados.

Goiânia se encontra em uma das condições mais preocupantes por ser a segunda no ranking de casos confirmados e ao mesmo tempo apresentar vulnerabilidade muito alta. Além de ser um polo de dispersão de casos, dada sua centralidade e influência sobre as demais cidades do interior do estado de Goiás, suas condições de desigualdade social implicam em pressões internas significativas.

Diante do cenário modelado para as condições socioeconômicas levantadas e o número de casos analisados, poderiam ser indicadas como prioritárias de atenção, a região metropolitana de Goiânia, o município de Anápolis e municípios limítrofes, o Distrito Federal e os municípios limítrofes, localizados na região conhecida como Entorno do DF. Medidas de controle mais efetivas nessas três regiões podem ser extremamente importantes para que os casos da doença não atinjam municípios ainda mais vulneráveis no Estado de Goiás.

\section{CONSIDERAÇÕES FINAIS}

Os determinantes sociais em saúde (condições socioeconômicas, culturais, ambientais, étnicoraciais, psicológicas e comportamentais) influenciam na qualidade de vida e no bem-estar das pessoas. Assim, estão diretamente relacionadas às vulnerabilidades sociais.

O IVSc elaborado para os municípios de Goiás e para o Distrito Federal revelou, a partir de sua classificação, as seguintes classes de vulnerabilidade: baixa, média, alta e muito alta. Para isso, foram levadas em conta disparidades quanto às principais variáveis socioeconômicas avaliadas, principalmente no que diz respeito à renda, condição de formalidade ou não da atividade remunerada exercida, saneamento básico apurado por meio da condição de abastecimento de água e as pressões dos setores de saúde, considerando o total de internações por doenças consideradas mais vulneráveis à COVID-19.

A maior parte das unidades territoriais avaliadas foi classificada em situação de média vulnerabilidade $(45 \%)$, seguidas de condições de baixa $(30 \%)$, alta $(15 \%)$ e muito alta vulnerabilidade $(8,5 \%)$. As melhores condições foram observadas para os municípios localizados na região Centro-Sul do Estado de Goiás, enquanto as piores encontram-se na região Nordeste do Estado.

No que se refere ao método, vale destacar que, ao escolher a escala municipal para as análises, peculiaridades internas dos municípios, ou seja, as desigualdades existentes em nível de bairro ou setor, não podem ser avaliadas, mas, reconhecendo-se tais condições seria interessante a modelagem do IVS $\mathrm{c}$ por setores censitários, começando a priori, pelas principais cidades polo dos casos de COVID-19 - Goiânia e Brasília.

Sabe-se, ainda, que, atualmente, encontram-se disponíveis inúmeros algoritmos para predição de variáveis e que vários poderiam ser avaliados quanto ao seu desempenho: regressão logística $(\mathrm{g} / \mathrm{m})$, regressão logística penalizada ( $g / m n e t)$, knn e os de redes neurais, apenas para citar alguns. Desta forma, seria interessante o desenvolvimento de mais pesquisas com o uso dos algoritmos de machine learning com essa finalidade, dado o potencial de aplicação já observado e a condição de gratuidade dos softwares disponíveis.

Por fim, ressalta-se que a pesquisa apresentada trata-se de um estudo experimental e que demanda revisões, avaliações críticas e validações de profissionais especialistas na área para efetiva aplicação, no entanto, quando comparado à pesquisa já realizada no Estado no que diz respeito às vulnerabilidades sociais, o IVS $\mathrm{c}$ apresentou bons resultados, o que indica a potencialidade do seu uso como instrumentos para planejamento de ações para o enfrentamento à COVID-19 no Estado de Goiás como no Distrito Federal.

\section{AGRADECIMENTOS}

A presente pesquisa foi realizada com apoio do Conselho Nacional de Desenvolvimento Científico e Tecnológico (CNPQ) - (Número do Processo: 141498/2019-6).

Agradecemos ao Professor Dr. Édipo Henrique Cremon - IFG Campus Goiânia, pelas orientações metodológicas, e ao Laboratório de Processamento de Imagens do Instituto de Estudos 
Socioambientais da UFG - LAPIG/IESA, pelo compartilhamento dos dados referentes aos registros de casos de COVID-19 no Estado de Goiás e no Distrito Federal.

\section{REFERÊNCIAS}

BRASIL, Ministério da Economia. RAIS - Relação Anual de Informações Sociais. Disponível em: http://www.rais.gov.br/sitio/index.jsf. Acesso em: 15 abr. 2020.

$\overline{\text { https://covid.saude.gov.br/. Acesso em: } 01 \text { maio. 2020. https://doi.org/10.15309/18psd190101 }}$

BREIMAN, L.; CUTLER. RandomForest: Random Forests for Classification and Regression, 2001. Disponível em: https://cran.r-project.org/web/packages/randomForest/index.html._Acesso em: 01 maio. 2020.

BUCCHIANERI, G. Is SARS a Poor Man's Disease? Socioeconomic Status and Risk Factors for SARS Transmission. Forum for Health Economics \& Policy, v.13,n.2, 2010. Disponível em http://dx.doi.org/10.2202/1558-9544.1209. Acesso em: 30 abr. 2020. https://doi.org/10.2202/1558$\underline{9544.1209}$

CHEN, T.; GUESTRIN, C. XGBoost: A Scalable Tree Boosting System. In: International Conference on Knowledge Discovery and Data Mining, 2016, San Francisco. Anais [...]. San Francisco: CA, 2016. Disponível em: https://doi.org/10.1145/2939672.2939785. Acesso em: 02 maio 2020. https://doi.org/10.1145/2939672.2939785

CZERESNIA, D.; OVIEDO, R. A. M. O conceito de vulnerabilidade e seu caráter biossocial. Interface Comunicação, saúde e Educação, v. 19, n. 53, p. 237-249, 2015. Disponível em: http://dx.doi.org/10.1590/1807-57622014.0436. Acesso em: 20 abr. 2020 . https://doi.org/10.1590/1807-57622014.0436

DATASUS - Departamento de Informática do SUS. Tabnet - Epidemiologia e Morbidade. Disponível em: https://datasus.saude.gov.br/informacoes-de-saude-tabnet/. Acesso em: 15 abr. 2020.

GRÖMPING, U. Variable importance in regression models. Wiley Interdisciplinary Reviews: Computational Statistics, v. 7, n. 2, p. 137-152, 2015. Disponível em: https://onlinelibrary.wiley.com/doi/abs/10.1002/wics.1346. Acesso em: 01 maio 2020. https://doi.org/10.1002/wics.1346

IBGE - Instituto Brasileiro de Geografia e Estatística. Cidades e Estados, 2019. Disponível em: https://www.ibge.gov.br/. Acesso em: 01 maio 2020.

Censo demográfico de 2010. Disponível em: https://sidra.ibge.gov.br/pesquisa/censodemografico/demografico-2010/inicial. Acesso em: 15 abr. 2020.

IMB - Instituto Mario Borges de Estatística e estudos socioeconômicos. A vulnerabilidade social dos municípios Goianos. Disponível em: https://www.imb.go.gov.br/. Acesso em: 01 maio 2020.

KUHN, M. Caret: classification and regression training. R package version 6.0-76; 2017. Disponível em: https://CRAN.R-project.org/package=caret. Acesso em: 20 abr. 2020.

LAPIG - Laboratório de Processamento de Imagens e Geoprocessamento. Plataforma COVID-19. Disponível em: https://covidgoias.ufg.br/. Acesso em: 15 abr. 2020.

OPAS - Organização Pan-Americana da Saúde. Folha Informativa - COVID-19. Disponível em: https://www.paho.org/bra/index.php?option=com_content\&view=article\&id=6101: covid19\&ltemid=875. Acesso em: 07 maio. 2020.

R- PROJECT. Disponível em: https://cran.r-project.org/. Acesso em: 10 jan. 2018.

QGIS. Disponível em: http://qgisbrasil.org/. Acesso em: 10 jan. 2018.

SANTANA, P. Ambientes e sujeitos sociais no mundo globalizado: a geografia da saúde e as demais áreas do conhecimento. In: OLIVEIRA, J. A.; SOUZA, G. A. (org.). Geografia da Saúde: ambientes e sujeitos sociais no mundo globalizado. Manaus: EDUA, 2016. p. 21-32.

SOHRABI, C.; et al. World Health Organization declares Global Emergency: A review of the 2019 Novel Coronavirus (COVID-19). International Journal of Surgery, vol. 76, p.71-76, apr. 2020. Disponível em: https://www.sciencedirect.com/science/article/pii/S1743919120301977?via\%3Dihub. Acesso em: 01 maio 2020. https://doi.org/10.1016/j.ijsu.2020.03.036 